\title{
Aplicación de las escalas de estratificación del riesgo en el diagnóstico de los síndromes coronarios agudos
}

\author{
John Jaime Sprockel Díaz ${ }^{\text {a,* }}$, Helio José Mantilla Durán ${ }^{a}$, \\ Lida Carolina Cruz Daza ${ }^{a}$, Rafael Andrés Barón ${ }^{a}$ y Juan José Diaztagle ${ }^{b}$
}

\footnotetext{
a Facultad de Medicina Interna, Fundación Universitaria de Ciencias de la Salud, Hospital de San José de Bogotá, Bogotá, Colombia

b Departamento de Ciencias Fisiológicas, Universidad Nacional de Colombia, Bogotá, Colombia
}

Recibido el 11 de agosto de 2016; aceptado el 6 de noviembre de 2016

Disponible en Internet el 5 de enero de 2017

\section{PALABRAS CLAVE \\ Dolor torácico; \\ Síndrome coronario agudo; \\ Infarto de miocardio; \\ Angina; \\ Diagnóstico}

\begin{abstract}
Resumen
Introducción: El dolor torácico es uno de los principales motivos de consulta en el servicio de urgencias. Diferentes escalas de riesgo han sido evaluadas en los pacientes con dolor torácico. El objetivo del presente estudio es comparar el desempeño diagnóstico de las escalas de riesgo en los pacientes con dolor torácico y sospecha de síndrome coronario agudo.

Métodos: Estudio de pruebas diagnósticas que incluyó pacientes mayores de 18 años con dolor torácico y sospecha de síndrome coronario agudo. El estándar de referencia fue alguna prueba de estratificación coronaria invasiva o no invasiva y la prueba índice la constituyó la puntuación de las escalas de riesgo TIMI, GRACE, CARdiac, Florencia, Sanchis y HEART. Se calcularon las características operativas para diferentes puntos de corte y se compararon las áreas bajo la curva ROC.

Resultados: Se incluyeron 249 pacientes, $143(57,4 \%)$ fueron de sexo masculino, 65,3 años de edad media; $73(29,3 \%)$ fueron anginas inestables, $79(31,7 \%)$ presentaron infartos agudos de miocardio sin elevación del ST y a 97 (39\%) se les descartó la enfermedad coronaria. Las escalas HEART y TIMI mostraron el mejor rendimiento diagnóstico con un área bajo la curva de 0,75 (IC del 95\% 0,69-0,81) y 0,71 (IC del 95\% 0,65-0,77). Las áreas bajo la curva de Florencia, CARdiac, GRACE, y Sanchis fueron 0,64 (IC 95\% 0,57-0,71), 0,63 (IC 95\% 0,57-0,69), 0,62 (IC 95\% 0,55-0,69), y 0,62 (IC 95\% 0,55-0,69), respectivamente.

Conclusiones: En una población de pacientes con alta probabilidad para el síndrome coronario agudo, las escalas HEART y TIMI mostraron una mayor capacidad para discriminar el diagnóstico del síndrome coronario agudo.

(C) 2016 Sociedad Colombiana de Cardiología y Cirugía Cardiovascular. Publicado por Elsevier España, S.L.U. Este es un artículo Open Access bajo la licencia CC BY-NC-ND (http:// creativecommons.org/licenses/by-nc-nd/4.0/).
\end{abstract}

\footnotetext{
* Autor para correspondencia.

Correo electrónico: jjsprockel@fucsalud.edu.co (J.J. Sprockel Díaz).
} 


\section{KEYWORDS}

Chest pain;

Acute coronary

syndrome;

Myocardial infarction; Angina;

Diagnosis
Implementation of risk stratification scales in the diagnosis of acute coronary syndromes

\begin{abstract}
Introduction: Chest pain is one of the main reasons for consultation in the emergency room. Several risk scales have been assessed in patients with chest pain. The motivation of this study is to compare the diagnostic performance of risk scales in patients with chest pain and suspicion of acute coronary syndrome.

Methods: Study of diagnostic tests that included patients over the age of 18 with chest pain and suspicion of acute coronary syndrome. The reference standard was an invasive or noninvasive coronary stratification test and the index test consisted of the score in risk scales TIMI, GRACE, CARdiac, Florencia, Sanchis and HEART. Operative characteristics for different cut points were calculated and the areas under the ROC curve were compared.

Results: The study included 249 patients, of whom 143 (57.4\%) were male, average age was 65.3 years; $73(29.3 \%)$ were unstable anginas; $79(31.7 \%)$ showed acute myocardial infarctions without ST elevation and for 97 (39\%) coronary disease was ruled out. HEART and TIMI scales revealed the best diagnostic performance with a low area under the curve of $0.75(\mathrm{Cl}$ of $95 \%$ 0.69-0.81) and 0.71 (Cl of 95\% 0.65-0.77). Areas under the curve for Florencia, CARdiac, GRACE, and Sanchis were 0,64 ( $\mathrm{Cl} 95 \% 0.57-0.71), 0.63$ ( $\mathrm{Cl} 95 \%$ 0.57-0.69), 0.62 ( $\mathrm{Cl} 95 \%$ 0.55-0.69), and 0.62 (Cl 95\% 0.55-0.69), respectively.

Conclusions: In a patient population with high probability for acute coronary syndrome, HEART and TIMI scales showed a greater ability to discriminate the diagnosis of acute coronary syndrome.

(c) 2016 Sociedad Colombiana de Cardiología y Cirugía Cardiovascular. Published by Elsevier España, S.L.U. This is an open access article under the CC BY-NC-ND license (http:// creativecommons.org/licenses/by-nc-nd/4.0/).
\end{abstract}

\section{Introducción}

El dolor torácico es uno de los principales motivos de consulta en el servicio de urgencias. Aunque la mayoría de las veces es causado por condiciones benignas, es también el principal síntoma de varias situaciones que ponen en riesgo la vida ${ }^{1}$, entre las que se encuentra el síndrome coronario agudo; la principal causa de muerte no trasmisible a nivel mundial ${ }^{2}$.

Los datos en Colombia indican que durante el año 2014 la primera causa de muerte, fueron las enfermedades isquémicas del corazón con 31.388 defunciones $(16,3 \%)^{3}$, esto conlleva a un alto consumo de recursos para el proceso diagnóstico del paciente con dolor torácico en búsqueda de un síndrome coronario agudo ${ }^{4}$.

La evaluación del dolor torácico por medio de la historia clínica presenta limitaciones importantes y se ha identificado que su desempeño puede ser pobre al momento del diagnóstico ${ }^{5}$. Además, cuando el seguimiento electrocardiográfico y los biomarcadores son negativos, se ha documentado una probabilidad de muerte o infarto inferior al $2 \% 6,7$, es de anotar que existen reportes mucho más altos, de un $3 \%$ a 30 días $^{8}$ y de $4,8 \%$ a 6 meses $^{9}$. Por lo tanto, todavía no es claro cuál es el mejor camino para la correcta y rápida estratificación de este grupo de pacientes.

En el año 1994 un grupo de cardiólogos encabezado por Braunwald recomendaron una forma de evaluar la probabilidad pretest para la enfermedad coronaria significativa en pacientes con síntomas sugestivos de angina inestable, basados en datos clínicos, electrocardiográficos y biomarcadores que por mucho tiempo fue recomendada y que en la actualidad ya no aparece en las guías de manejo ${ }^{10}$. Luego de este enfoque, el esfuerzo se ha centrado en el desarrollo y aplicación de las escalas pronósticas, algunas de ellas inicialmente desarrolladas para la estratificación del riesgo de eventos cardiovasculares mayores en los síndromes coronarios agudos ya diagnosticados, por ejemplo: TIMI, GRACE y otras escalas directamente elaboradas sobre poblaciones de pacientes con dolor torácico de bajo riesgo para el síndrome coronario agudo (CARdiac, Florencia y Sanchis) ${ }^{11-13}$, así como en la población general con dolor torácico (HEART) ${ }^{14}$.

El pronunciamiento que hacen las guías colombianas de manejo del síndrome coronario agudo sin elevación del ST acerca del uso de estas escalas en la evaluación inicial del dolor torácico indica que: "usar un modelo para estratificación de riesgo como el TIMI o puntaje de riesgo GRACE o PURSUIT, puede ser útil para tomar una decisión con relación a las opciones de tratamiento en pacientes con sospecha de síndrome coronario agudo', (Ila-B $)^{15}$. Mientras que las guías del síndrome coronario agudo con elevación del ST recomiendan que: "en los pacientes que consultan por dolor torácico y sospecha diagnóstica de síndrome coronario agudo, luego de la determinación rápida de la probabilidad se realiza una estratificación de riesgo basado en: los síntomas, el examen físico, los cambios electrocardiográficos y los marcadores bioquímicos'" $(\mathrm{I}-\mathrm{B})^{16}$.

La American Heart Association también fija una posición con respecto a los sistemas de estratificación del riesgo aplicados a los pacientes con dolor torácico de baja 
probabilidad: "... son recomendados como guía para la estratificación del riesgo y se deben aplicar como complemento, no como substitutos, del juicio clínico en la evaluación de los pacientes que presentan dolor en el pecho" "17. Ante la falta de claridad acerca de la mejor forma de realizar el enfoque clínico inicial de los pacientes con dolor torácico y sospecha de síndrome coronario agudo, en particular para aquellos pacientes con angina inestable, las escalas de riesgo representan un camino prometedor en el proceso diagnóstico y de toma de decisiones.

En la actualidad, contando con la troponina de alta sensibilidad, la evaluación del dolor torácico puede realizarse siguiendo varios protocolos: $0 \mathrm{~h} / 1 \mathrm{~h}$ y $0 \mathrm{~h} / 3 \mathrm{~h}$, troponina más cotinina y aquellos basados en las escalas (por ejemplo: TIMI en el ADAPT, HEART, EDCAST, entre otras) ${ }^{18}$. El protocolo $0 \mathrm{~h} / 3 \mathrm{~h}$ y los últimos mencionados se basan en el resultado de diferentes escalas, en los últimos años la investigación se ha encaminado a identificar cuál de estas logra una mejor capacidad de discriminación diagnóstica o que por lo menos permita dar un alta temprana segura. Es por ello que el objetivo del presente trabajo es realizar un estudio de pruebas diagnósticas, comparando el desempeño entre las diferentes escalas de riesgo de eventos cardiovasculares mayores, en el diagnóstico de los síndromes coronarios agudos en una población de pacientes con dolor torácico y sospecha del síndrome coronario agudo sin elevación del ST.

\section{Metodología}

Se realizó un estudio de pruebas diagnósticas tomando como referencia una cohorte histórica, en la cual se incluyeron de manera consecutiva pacientes mayores de 18 años de edad que ingresaron al servicio de urgencias o a la unidad de cuidado coronario por dolor torácico con sospecha del síndrome coronario agudo sin elevación del segmento ST, en el Hospital de San José y Hospital Infantil Universitario de San José, dos centros de tercer nivel de atención en Bogotá, entre el 1 de agosto de 2013 y el 1 de noviembre de 2015 . No se calculó a priori el tamaño de la muestra; por lo que se decidió calcular la potencia estadística con el método de comparación de pruebas diagnósticas de grupos pareados.

El diagnóstico se basó en el seguimiento clínico, electrocardiográfico y de biomarcadores (troponina ultrasensible por método PATHFAST de Mitsubishi Company, Japón) de 12 a 24 horas en el servicio de urgencias seguido por la estratificación coronaria. El estándar de referencia para el diagnóstico de infarto agudo de miocardio, estuvo basado en la tercera redefinición $^{19}$, y para el caso de la angina inestable de alto riesgo se basó en la presencia de datos clínicos compatibles (dolor torácico de reciente comienzo, in crescendo o en reposo), junto con la positividad en alguna estrategia de estratificación coronaria invasiva o no invasiva ${ }^{20}$. En algunos casos se pudo disponer de una prueba inequívoca que confirmara la presencia de una condición que explicara el origen del dolor torácico, como el angiotac en el caso de un embolismo pulmonar o de una disección aortica, descartándose de esta forma el síndrome coronario agudo. El diagnóstico fue establecido durante el tiempo de la hospitalización por un cardiólogo, un internista o un intensivista.

La prueba índice, estuvo constituida por la puntuación de cada una de las escalas de riesgo: TIMI, HEART, GRACE,
CARdiac, Florencia y Sanchis. Los investigadores calcularon la puntuación de cada una de las escalas a todos los pacientes a partir de los datos recolectados en un momento posterior al de la hospitalización, de manera que no afectó la toma de decisiones en el manejo de los pacientes.

Se recolectaron las características demográficas de la población, presentación clínica, antecedente de la enfermedad coronaria o la enfermedad renal crónica definidas como hallazgo en el historial clínico documentado por el servicio de medicina interna, alteraciones del electrocardiograma, pruebas de laboratorio, el resultado de cada una de las escalas y el resultado de la estratificación coronaria de los pacientes incluidos.

Para el análisis de la información se construyó una base de datos en Excel 2010. El análisis estadístico se realizó en Stata $13^{\circledR}$. Las variables categóricas se expresan con frecuencias absolutas y relativas, las cuantitativas con medidas de tendencia central y dispersión. Los valores $p$ representan la probabilidad de que las proporciones (prueba de jí cuadrado) o las medianas (prueba de $U$ de Mann Whitney) sean iguales en ambos grupos. Mediante las tablas de contingencia $(2 \times 2)$ de cada una de las escalas de riesgo, se tomaron los diferentes puntos de corte, calculando: la sensibilidad, la especificidad, la precisión y las razones de verosimilitud (likelyhood ratio). Se muestran las áreas bajo la curva ROC no paramétrica de cada una de las escalas evaluadas, sus errores estándar y se compararon entre sí mediante el test de igualdad de curvas ROC, con un nivel de significancia menor a 0,003 utilizando el método sugerido por Delong y Clarke Pearson ${ }^{21}$ y haciendo el ajuste de Bonferroni para las comparaciones múltiples.

El estudio fue aprobado por el comité de investigaciones y el comité de ética en investigación con seres humanos de la Facultad de Medicina de la Fundación Universitaria Ciencias de la Salud y el Hospital de San José de Bogotá.

\section{Resultados}

\section{Descripción general de la cohorte}

Durante los quince meses del estudio se lograron recolectar 249 pacientes, de los cuales $143(57,4 \%)$ fueron de sexo masculino, con un promedio de edad de 65,3 años con una desviación estándar de 12,9. El diagnóstico final fue angina inestable de alto riesgo en 73 pacientes $(29,3 \%)$, infarto agudo de miocardio sin elevación del ST en 79 pacientes $(31,7 \%)$ y a 97 pacientes se les descartó síndrome coronario agudo (39\%). Del total de la población, se realizó coronariografía en 142 casos (57\%), a 67 (27\%) otro método de estratificación no invasiva (perfusión miocárdica o ecocardiografía estrés con dobutamina) y a 40 casos (16\%) se utilizó el criterio clínico más otras pruebas, por ejemplo: la gammagrafía de ventilación y perfusión, la tomografía de tórax con protocolo para embolismo pulmonar, para diagnosticar la causa del dolor torácico.

La distribución de las comorbilidades indicó que 63 pacientes $(25,3 \%)$ presentaban diabetes mellitus tipo 2,33 $(13,2 \%)$ enfermedad renal crónica y $103(41,3 \%)$ tenían antecedente de enfermedad coronaria. De acuerdo a la escala de probabilidad pretest propuesta por Braunwald, 216 (86,7\%) tenían dolor torácico de alta probabilidad y $33(13,3 \%)$ tenían 
Tabla 1 Características demográficas de la población

\begin{tabular}{llll}
\hline Variable & Sin SCA $n=97$ & Con SCA $n=152$ & Valor $\mathrm{p}$ \\
\hline Edad (años), promedio (DE) & $63(50-74)$ & $66(56-77)$ & 0,06 \\
Mayores de 75 años, $\mathrm{n}(\%)$ & $24(24,74)$ & $45(29,61)$ & 0,40 \\
Sexo masculino, $\mathrm{n}(\%)$ & $51(52,58)$ & $92(60,53)$ & 0,22 \\
Diabetes Mellitus 2, $\mathrm{n}(\%)$ & $18(18,56)$ & $45(29,61)$ & 0,05 \\
Antecedente de Enfermedad Renal Crónica* $\mathrm{n}(\%)$ & $9(9,28)$ & $24(15,79)$ & 0,14 \\
Antecedente de Enfermedad Coronaria, $\mathrm{n}(\%)$ & $26(26,80)$ & $77(50,66)$ & $<0,001$ \\
\hline
\end{tabular}

SCA: Síndrome coronario agudo.

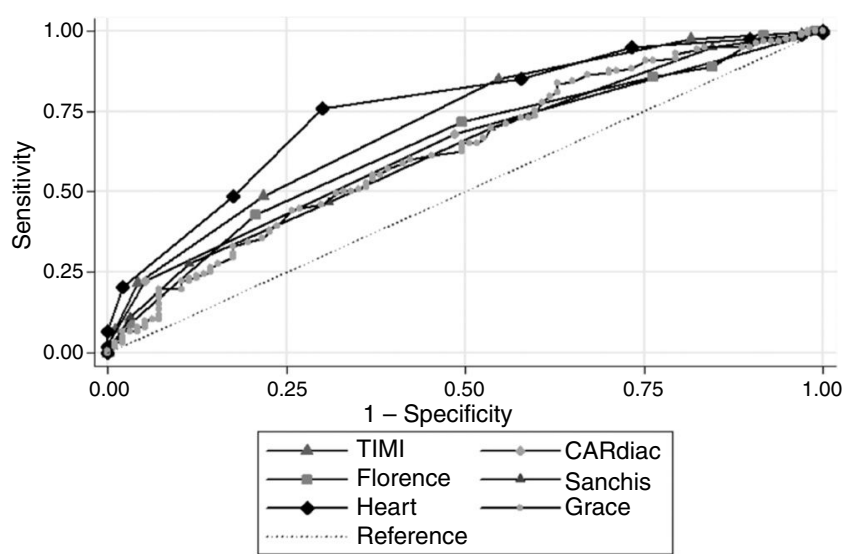

Figura 1 Áreas bajo las curvas ROC de HEART, TIMI, Florencia, CARdiac, GRACE y Sanchis.

probabilidad intermedia; no hubo pacientes de baja probabilidad. Al comparar las características de los pacientes que tuvieron síndrome coronario agudo con los que no lo tuvieron, hubo una mayor proporción de antecedente de la enfermedad coronaria y de la diabetes, con una tendencia a ser mayores de edad (tabla 1).

\section{Comparación entre las escalas y el diagnóstico}

El área bajo la curva ROC de las escalas TIMI, HEART, GRACE, CARdiac, Florencia y Sanchis se presenta en la tabla 2 y en la fig. 1, en donde se observa que la escala HEART presentó la mejor capacidad de discriminación diagnóstica para los síndromes coronarios agudos con un área bajo la curva de 0,75 (IC del 95\% 0,69-0,81); el mejor punto de corte fue una puntuación mayor o igual a 6 , con una sensibilidad del $75,6 \%$, una especificidad del $70,1 \%$ y una precisión del $73,5 \%$ (tabla 3). La mejor especificidad de la escala se encontró con un puntaje mayor o igual a 8 , con una especificidad del $97,9 \%$ y $\mathrm{LR}^{+}$de 9,9 .

La escala de TIMI obtuvo un área bajo la curva de 0,71 (IC 95\% 0,65-0,77), el mejor punto de corte fue con un puntaje mayor o igual a 2 puntos, con una sensibilidad del $84,8 \%$, una especificidad del $45,4 \%$ y una precisión del $69,5 \%$ (tabla 4). Con un puntaje mayor o igual a 4 puntos en la escala, se encontró una especificidad del 95,5\% con un $\mathrm{LR}^{+}$ de 5,2 .

La escala de Florencia obtuvo un área bajo la curva de 0,64 (IC 95\% 0,57-0,71), clasificando correctamente al $63,4 \%$ de los pacientes con un puntaje mayor o igual a 5 , con una sensibilidad del $71,7 \%$ y una especificidad del $50,5 \%$ (tabla 5). El área bajo la curva obtenida para CARdiac fue de 0,63 (IC 95\% 0,57-0,69), clasificando correctamente al $61,4 \%$ de los pacientes con un puntaje mayor o igual a 1 , con una sensibilidad del $67,7 \%$ y una especificidad del $51,5 \%$ (tabla 6).

Las escalas de GRACE y Sanchis obtuvieron un área bajo la curva de 0,62 (IC 95\% 0,55-0,69). El punto de mejor discriminación para el GRACE fue a los 89 puntos con una sensibilidad del $86,2 \%$ y una especificidad del $33 \%$. Para el caso de Sanchis se clasificaba correctamente al $63,9 \%$ con un puntaje mayor o igual a 1, logrando una sensibilidad del $94,7 \%$ y una especificidad del $15,5 \%$ (tabla 7 ).

Al realizar la comparación de las áreas bajo las curvas ROC, no encontramos una diferencia estadísticamente significativa entre las escalas HEART vs. TIMI $(p=0,11)$, pero sí entre las de HEART y GRACE $(p<0,001)$, así como entre las de TIMI y GRACE $(p=0,02)$.

Con base en los maximos valores de sensibilidad y especificidad obtenidos para la escala de HEART y con una

Tabla 2 Áreas bajo las curvas ROC (AUC) de las escalas evaluadas con sus intervalos de confianza (IC), errores estándar (EE) y precisión según el mejor punto de corte

\begin{tabular}{|c|c|c|c|c|c|}
\hline Escala & AUC & $\mathrm{EE}$ & [IC 95\%] & Punto de corte & Precisión \% \\
\hline HEART & 0,75 & 0,030 & $0,69-0,81$ & 6 & 73,4 \\
\hline TIMI & 0,71 & 0,031 & $0,65-0,77$ & 2 & 69,4 \\
\hline FLORENCIA & 0,64 & 0,034 & $0,57-0,71$ & 5 & 63,4 \\
\hline CARdiac & 0,63 & 0,032 & $0,57-0,69$ & 1 & 61,4 \\
\hline GRACE & 0,62 & 0,036 & $0,55-0,69$ & 89 & 65,4 \\
\hline SANCHIS & 0,62 & 0,035 & $0,55-0,69$ & 1 & 63,9 \\
\hline
\end{tabular}


Tabla 3 Características operativas de la escala de HEART discriminada de acuerdo a los diferentes puntos de corte

\begin{tabular}{|c|c|c|c|c|c|}
\hline Punto de Corte & Sensibilidad \% & Especificidad \% & Precisión \% & $\mathrm{LR}^{+}$ & $\mathrm{LR}^{-}$ \\
\hline 0 & 100 & 0 & 61 & 1 & \\
\hline 1 & 99,3 & 0 & 60,6 & 0,9 & \\
\hline 2 & 98,6 & 3 & 61,4 & 1 & 0,4 \\
\hline 3 & 97,3 & 10,3 & 63,4 & 1 & 0,2 \\
\hline 4 & 94,7 & 26,8 & 68,2 & 1,2 & 0,1 \\
\hline 5 & 84,8 & 42,2 & 68,2 & 1,4 & 0,3 \\
\hline 6 & 75,6 & 70,1 & 73,4 & 2,5 & 0,3 \\
\hline 7 & 48,6 & 82,4 & 61,8 & 2,7 & 0,6 \\
\hline 8 & 20,3 & 97,9 & 50,6 & 9,8 & 0,8 \\
\hline 9 & 6,5 & 100 & 42,9 & & 0,9 \\
\hline 10 & 1,9 & 100 & 40,1 & & 0,9 \\
\hline
\end{tabular}

Tabla 4 Características operativas de la escala de TIMI discriminada de acuerdo a los diferentes puntos de corte

\begin{tabular}{lcclcr}
\hline Punto de corte & Sensibilidad \% & Especificidad \% & Precisión \% & $\mathrm{LR}^{+}$ & $\mathrm{LR}^{-}$ \\
\hline 0 & 100 & 0 & 61 & 1 & 1,1 \\
1 & 97,3 & 18,5 & 66,6 & 0,1 \\
2 & 84,8 & 45,3 & 69,4 & 0,3 \\
3 & 48,6 & 78,3 & 60,2 & 2,2 & 0,6 \\
4 & 21,7 & 95,8 & 50,6 & 5,2 & 0,8 \\
5 & 7,2 & 98,9 & 42,9 & 7 & 0,9 \\
6 & 1,9 & 100 & 40,1 & & 0,9 \\
\hline
\end{tabular}

Tabla 5 Características operativas de la escala de Florencia discriminadas de acuerdo a los diferentes puntos de corte

\begin{tabular}{|c|c|c|c|c|c|}
\hline Punto de corte & Sensibilidad \% & Especificidad \% & Precisión \% & $\mathrm{LR}^{+}$ & $\mathrm{LR}^{-}$ \\
\hline 0 & 100 & 0 & 61 & 1 & \\
\hline 1 & 100 & 1 & 61,4 & 1 & 0 \\
\hline 2 & 98,6 & 8,2 & 63,4 & 1 & 0,1 \\
\hline 3 & 88,8 & 15,4 & 60,2 & 1 & 0,7 \\
\hline 4 & 85,5 & 23,7 & 61,4 & 1,1 & 0,6 \\
\hline 5 & 71,7 & 50,5 & 63,4 & 1,4 & 0,5 \\
\hline 6 & 42,7 & 79,3 & 57,0 & 2 & 0,7 \\
\hline 7 & 9,2 & 96,9 & 43,3 & 2,9 & 0,9 \\
\hline
\end{tabular}

Tabla 6 Características operativas de la escala de CARdiac discriminadas de acuerdo a los diferentes puntos de corte

\begin{tabular}{lcclrl}
\hline Punto de corte & Sensibilidad $\%$ & Especificidad \% & Precisión \% & LR $^{+}$ & LR $^{-}$ \\
\hline 0 & 100 & 0 & 61 & 1 & 1,3 \\
1 & 67,7 & 51,5 & 61,4 & 0,6 \\
2 & 22,3 & 94,8 & 50,6 & 4,3 & 0,8 \\
3 & 2,6 & 100 & 40,5 & & 0,9 \\
\hline
\end{tabular}

Tabla 7 Características operativas de la escala de Sanchis discriminadas de acuerdo a los diferentes puntos de corte

\begin{tabular}{|c|c|c|c|c|c|}
\hline Punto de corte & Sensibilidad \% & Especificidad \% & Precisión \% & $\mathrm{LR}^{+}$ & $\mathrm{LR}^{-}$ \\
\hline 0 & 100 & 0 & 61 & 1 & \\
\hline 1 & 94,7 & 15,4 & 63,8 & 1,1 & 0,3 \\
\hline 2 & 69,7 & 46,3 & 60,6 & 1,3 & 0,6 \\
\hline 3 & 46,7 & 69 & 55,4 & 1,5 & 0,7 \\
\hline 4 & 27,6 & 88,6 & 51,4 & 2,4 & 0,8 \\
\hline 5 & 11,1 & 96,9 & 44,5 & 3,6 & 0,9 \\
\hline 6 & 0,6 & 100 & 39,3 & & 0,9 \\
\hline
\end{tabular}


prevalencia de la enfermedad del $61 \%$, un tamaño de muestra final de 249 pacientes, se calculó una potencia estadística del $51,6 \%$ con base en la sensibilidad y del $93,6 \%$ con base en la especificidad.

\section{Discusión}

Las escalas de estratificación de riesgo en el síndrome coronario agudo más reconocidas a nivel internacional son la de TIMI y GRACE. La escala de riesgo de TIMI en el síndrome coronario agudo sin elevación del segmento ST, se comenzó a evaluar en los servicios de urgencias en los pacientes con dolor torácico indiferenciado poco tiempo después de su desarrollo y validación. Bartholomew y Cols., encontraron un aumento del riesgo de eventos adversos coronarios mayores por cada punto de elevación en la escala de TIMI a 30 días $^{22}$, mientras otros estudios documentaron que el riesgo no desaparece cuando la puntuación es de cero ${ }^{23,24}$. Posteriormente, la escala de GRACE fue evaluada con el mismo propósito por Lyon y Cols., en un estudio en donde la compara con TIMl y obtiene resultados similares a los ya descritos con un área bajo la curva de 0,80 y 0,79 , respectivamente ${ }^{25}$. Otro estudio documentó que el GRACE sobreestima el riesgo en los pacientes sanos ${ }^{26}$, mientras que un trabajo reciente concluye que ninguna de las dos escalas podía discriminar con precisión aquellos que tendrían un desenlace adverso cardiovascular mayor a 30 y 90 días $^{27}$.

Ante las limitaciones presentadas por las dos escalas para el diagnóstico del síndrome coronario agudo, varios grupos desarrollaron otras formas de evaluar el dolor torácico. Las escalas CARdiac ${ }^{11}$, Florencia $^{12}$, Sanchis ${ }^{13}$ y HEART ${ }^{14}$ forman parte de este grupo. Entre estas, la escala HEART ha sido la que más se ha evaluado desde su desarrollo en el año 2008, y en múltiples estudios, se alcanzan mejores resultados que con las escalas TIMI y GRACE ${ }^{28-30}$, aunque también se conoce un caso de reporte negativo ${ }^{31}$. Estos estudios han evaluado la capacidad pronóstica de estas escalas.

La evaluación del dolor torácico ha encontrado en las escalas de riesgo una forma para mejorar la toma de decisiones. Desde este punto de vista se vislumbra una relación entre el diagnóstico y el pronóstico, ya que se puede asumir que este último es peor en aquellos casos en los que se confirme la presencia de un síndrome coronario agudo. Nuestro estudio se centró en la capacidad diagnóstica de estas escalas, lo cual se convierte en un punto diferenciador frente a los trabajos ya discutidos. En este sentido encontramos dos trabajos que tuvieron un enfoque similar al nuestro, por un lado Fersmire y Cols., evaluaron las escalas HEART y HEARTS3 considerando como desenlace el diagnóstico de infarto intrahospitalario o del síndrome coronario agudo a 30 días $^{32}$, y por otro lado, Conti y Cols., compararon varias escalas incluyendo la presencia de obstrucción coronaria documentada por cateterismo, el diagnóstico de angina o infarto junto con los otros desenlaces cardiovasculares mayores ${ }^{33}$. En ambos casos se encontró una buena capacidad discriminativa, en especial en el primer caso, con un resultado similar al presente reporte.

En el presente estudio se obtuvo un mejor resultado para la escala de HEART, situándose no muy lejos de la escala de TIMI estando superpuestos los intervalos de confianza. Se puede considerar que estas dos escalas poseen un poder de discriminación diagnóstica suficiente, que permita su aplicación clínica en los dolores torácicos. Debe anotarse que, de manera similar a lo encontrado en la bibliografía consultada ${ }^{34}$, en este estudio, con excepción de la escala de Florencia, un resultado negativo no puede descartar con certeza la presencia de un síndrome coronario agudo (resultados no presentados). Por otro lado, para cada una de las escalas se encuentra un punto para el cual la especificidad es muy alta (superior al $95 \%$ ) con lo que casi se podría dar por confirmado el diagnóstico de un síndrome coronario agudo.

Desde un punto de vista práctico, para el clínico en urgencias, puede ser más importante poder llegar a realizar el diagnóstico adecuado que tener un resultado de un riesgo potencial a un determinado tiempo. El uso del pronóstico en el abordaje del dolor torácico puede tener la limitación de que los desenlaces, eventualmente, puedan ser debidos a otros diagnósticos diferentes al síndrome coronario agudo. No obstante, definir una población en la cual se haya establecido un diagnóstico definitivo del síndrome coronario agudo reviste cierto grado de dificultad ya que el estándar de referencia no es perfecto, como ejemplo de ello está el hecho de que tener una angiografía negativa no descarta por completo la enfermedad coronaria y una perfusión miocárdica en enfermedad multivaso puede ser negativa. Esto hace que sea importante el seguimiento del paciente (a treinta días o a mayor tiempo) dentro de los estudios de evaluación del dolor torácico.

Dado que no todos los dolores torácicos son sometidos a una evaluación diagnóstica intrahospitalaria, nuestro estudio tiene el sesgo de seleccionar una población de alta probabilidad (sólo el $39 \%$ fueron negativos) con lo que los resultados no pueden generalizarse a todos los dolores torácicos, ya que la población evaluada puede influir en los resultados del desempeño diagnóstico de cada una de las escalas. Como ejemplo de este comportamiento diferencial tenemos que en un trabajo previo realizado por nuestro grupo en una población de pacientes con dolor torácico de baja probabilidad, las escalas TIMI y GRACE no discriminaron el diagnóstico de los síndromes coronarios agudos (área bajo la curva 0,54 y 0,51 , respectivamente) $)^{35}$. Será tema de otro estudio la aplicación de estas escalas en la población con dolor torácico sugestivo del síndrome coronario agudo, el electrocardiograma normal y la troponina negativa.

Ante estos resultados se debe reconocer que no se puede pretender llegar en todos los casos, en particular para la angina inestable, a un diagnóstico definitivo del síndrome coronario agudo solamente con una escala que contiene datos clínicos, electrocardiográficos y de laboratorio, sin contar con alguna estrategia adicional de estratificación coronaria. Estas escalas son una herramienta adicional en la identificación de dicha entidad y ofrecen un soporte más al clínico al momento de tomar decisiones. Como se concluye en un metaanálisis de estas herramientas, no deberían utilizarse como único medio para determinar la conducta médica, sino como un complemento a la apreciación clínica ${ }^{34}$.

Dentro de las limitaciones del presente trabajo debemos mencionar, además, que el tamaño de la muestra se fijó por conveniencia debido a que se aprovechó una base de datos que se tuvo disponible. La evaluación del dolor torácico es un problema complejo y costoso, al no poder disponer del referente estándar ideal, en este caso el cateterismo cardiaco en 
todos los casos, tomamos como referente una combinación de estudios aceptados internacionalmente y confirmados por especialistas del tema.

\section{Conclusiones}

En una población de pacientes con alta probabilidad para el síndrome coronario agudo, las escalas HEART y TIMI mostraron una adecuada capacidad para discriminar el diagnóstico del síndrome coronario agudo. Un puntaje de 0 en las escalas evaluadas y GRACE con puntajes de bajo riesgo (1-108) no descartó la presencia del síndrome coronario agudo con excepción de la escala de Florencia.

\section{Responsabilidades éticas}

Protección de personas y animales. Los autores declaran que para esta investigación no se han realizado experimentos en seres humanos ni en animales.

Confidencialidad de los datos. Los autores declaran que han seguido los protocolos de su centro de trabajo sobre la publicación de datos de pacientes.

Derecho a la privacidad y consentimiento informado. Los autores declaran que en este artículo no aparecen datos de pacientes.

\section{Conflicto de intereses}

Ninguno.

\section{Bibliografía}

1. Erhardt L, Herlitz J, Bossaert L, Halinen M, Keltai M, Koster R, et al. Task force on the management of chest pain. Eur Heart J. 2002 Aug;23:1153-76.

2. WHO. I Deaths from CVD and diabetes [Internet]. WHO. [cited 2013 Feb 27]. Available from: http://www.who.int/gho/ ncd/mortality_morbidity/cvd/en/index.html

3. Departamento Administrativo Nacional de Estadística, DANE. Defunciones no fetales 2014 - preliminar: Cuadro 11. Defunciones por grupos de edad y sexo, según departamento de ocurrencia y grupos de causas de defunción (lista Colombia 105 para la tabulación de mortalidad) [Internet]. DANE. 2015 [cited 2015 Jun 15]. Available from: http://www.dane.gov.co/files/ investigaciones/poblacion/defunciones/defun_2014/Cuadro11_ mar31.xls

4. Tosteson AN, Goldman L, Udvarhelyi IS, Lee TH. Costeffectiveness of a coronary care unit versus an intermediate care unit for emergency department patients with chest pain. Circulation. 1996;94:143-50.

5. Swap CJ, Nagurney JT. Value and Limitations of Chest Pain History in the Evaluation of Patients With Suspected Acute Coronary Syndromes. JAMA. 2005 Nov 23;294:2623-9.

6. Hamm WC, Braunwald E. A classification of unstable angina revisited. Circulation. 2000;102:118-22.

7. Lee TH, Goldman L. Evaluation of the patient with acute chest pain. N Engl J Med. 2000;342:1187-95.

8. Newby LK, Storrow AB, Gibler WB, Garvey JL, Tucker JF, Kaplan $\mathrm{AL}$, et al. Bedside multimarker testing of risk stratification in chest pain units. The chest pain evaluation by creatine
kinase-MB, myoglobin and troponin I (CHECKMATE) study. Circulation. 2001;103:1832-7.

9. Sanchis J, Bodí V, Llácer A, Núñez J, Consuegra L, Bosch MJ, et al. Risk stratification of patients with acute chest pain and normal troponin concentrations. Heart. 2005;91:1013-8.

10. Braunwald E, Jones RH, Mark DB, Brown J, Brown L, Cheitlin $M D$, et al. Diagnosing and managing unstable angina. Agency for Health Care Policy and Research. Circulation. 1994;90:613-22.

11. Fuller M, Hamilton D, Holly J, Mallin M, Rayner T, Eshenroder N, et al. Prospective Evaluation of a Simplified Risk Stratification Tool for Patients With Chest Pain in an Emergency Department Observation Unit. Crit Path Cardiol. 2013;12:132-6.

12. Conti A, Vanni S, Taglia BD, Paladini B, Magazzini S, Grifoni $S$, et al. A new simple risk score in patients with acute chest pain without existing known coronary disease. Am J Emerg Med. 2010;28:135-42.

13. Sanchis J, Bodí V, Núñez J, Bertomeu-González V, Gómez C, Bosch MJ, et al. New risk score for patients with acute chest pain, non-ST-segment deviation, and normal troponin concentrations: a comparison with the TIMI risk score. J Am Coll Cardiol. 2005;46:443-9.

14. Six AJ, Backus BE, Kelder JC. Chest pain in the emergency room: value of the HEART score. Neth Heart J. 2008;16:191-6.

15. Beltrán J, Beltrán R, Caicedo V, García M, García E, Gómez E, et al. Guías colombianas de cardiología Síndrome coronario agudo sin elevación del ST: Puntajes de riesgo en pacientes con síndrome coronario agudo. Rev Col Cardiol. 2008;15 Supl 3:170-4.

16. Beltrán J, Beltrán R, Bohórquez R, Diez JG, García C, García $\mathrm{E}$, et al. Guías colombianas de cardiología Síndrome coronario agudo con elevación del ST: Estratificación del riesgo en pacientes con síndrome coronario agudo con elevación del ST. Rev Col Cardiol. 2010;17 Supl 3:156-9.

17. Amsterdam EA, Kirk JD, Bluemke DA, Diercks D, Farkouh ME, Garvey JL, et al. Testing of Low-Risk Patients Presenting to the Emergency Department With Chest Pain. Circulation. 2010 Oct 26;122:1756-76.

18. Roffi M, Patrono C, Collet J, Mueller C, Valgimigli M, Andreotti F, et al. 2015 ESC Guidelines for the management of acute coronary syndromes in patients presenting without persistent ST-segment elevation: Task force for the management of acute coronary syndromes in patients presenting without persistent ST - Segment Elevation of the European Society of Cardiology (ESC). Eur Heart J. 2016;37:267-315.

19. Thygesen K, Alpert JS, Jaffe AS, Simoons ML, Chaitman BR, White HD, et al. Third universal definition of myocardial infarction. Circulation. 2012 Oct 16;126:2020-35.

20. Hamm CW, Bassand J-P, Agewall S, Bax J, Boersma E, Bueno $\mathrm{H}$, et al. ESC Guidelines for the management of acute coronary syndromes in patients presenting without persistent ST-segment elevation: The task force for the management of acute coronary syndromes (ACS) in patients presenting without persistent STsegment elevation of the European Society of Cardiology (ESC). Eur Heart J. 2011 Aug 26;32:2999-3054.

21. DeLong ER, DeLong DM, Clarke-Pearson DL. Comparing the areas under two or more correlated receiver operating characteristic curves: a nonparametric approach. Biometrics. 1988;44:837-45.

22. Bartholomew BA, Sheps DS, Monroe S, Mc Gorray S, Smith K, Pepine CJ. A Population-Based Evaluation of the Thrombolysis in Myocardial Infarction Risk Score for Unstable Angina and Non-ST Elevation Myocardial Infarction. Clin Cardiol. 2004;27: 74-8.

23. Pollack CV Jr, Sites FD, Shofer FS, Sease KL, Hollander JE. Application of the TIMI risk score for unstable angina and non-ST elevation acute coronary syndrome to an unselected emergency department chest pain population. Acad Emerg Med. 2006;13:13-8. 
24. Macdonald SP, Nagree Y, Fatovich DM, Flavell HL, Loutsky F. Comparison of two clinical scoring systems for emergency department risk stratification of suspected acute coronary syndrome. Emerg Med Australas. 2011;23:717-25.

25. Lyon R, Morris AC, Caesar D, Gray S, Gray A. Chest pain presenting to the Emergency Department-to stratify risk with GRACE or TIMI? Resuscitation. 2007;74:90-3.

26. Lee B, Chang AM, Matsuura AC, Marcoon S, Hollander JE. Comparison of cardiac risk scores in ED patients with potential acute coronary syndrome. Crit Path Cardiol. 2011;10:64-8.

27. Goodacre SW, Bradburn M, Mohamed A, Gray A. Evaluation of global registry of acute cardiac events and thrombolysis in myocardial infarction scores in patients with suspected acute coronary syndrome. Am J Emerg Med. 2012;30:37-44.

28. Melki D, Jernberg T. HEART Score A simple and useful tool that may lower the proportion of chest pain patients who are admitted. Crit Path Cardiol. 2013;12:127-31.

29. Six AJ, Cullen L, Backus BE, Greenslade J, Parsonage W, Aldous $\mathrm{S}$, et al. The HEART score for the assessment of patients with chest pain in the emergency department: A multinational validation study. Crit Path Cardiol. 2013;12:121-6.

30. Backus BE, Six AJ, Kelder JC, Bosschaert MAR, Mast EG, Mosterd $A$, et al. A prospective validation of the HEART score for chest pain patients at the emergency department. Intern J Cardiol. 2013;168:2153-8

31. Visser A, Wolthuis A, Breedveld R, ter Avest E. HEART score and clinical gestalt have similar diagnostic accuracy for diagnosing ACS in an unselected population of patients with chest pain presenting in the ED. Emerg Med J. 2015;32(8): 595-600.

32. Fesmire FM, Martin EJ, Cao Y, Heath GW. Improving risk stratification in patients with chest pain: the Erlanger HEARTS3 score. Am J Emerg Med. 2012;30:1829-37.

33. Conti A, Poggioni C, Viviani G, Mariannini Y, Luzzi M, Cerini G, et al. Risk scores prognostic implementation in patients with chest pain and nondiagnostic electrocardiograms. Am J Emerg Med. 2012;30:1719-28.

34. Hess EP, Agarwal D, Chandra S, Murad MH, Erwin PJ, Hollander JE, et al. Diagnostic accuracy of the TIMI risk score in patients with chest pain in the emergency department: a meta-analysis. CMAJ. 2010;182:1039-44.

35. Sprockel J, Barón R, Múñoz L, Peña M, Torres J. Comparación de escalas de riesgo en el diagnóstico del síndrome coronario agudo en pacientes con dolor torácico. Acta Med Colomb. 2014;39 2 supl. 1:15. 